\title{
The impact of the Internet on academic research practices
}

\author{
C. Dowling \\ Australian Catholic University \\ 412 Mt Alexander Rd, Ascot Vale, Victoria 3032, Australia, \\ e-mail: c.dowling@mercy.acu.edu.au
}

\begin{abstract}
This paper discusses some aspects of the use of the Internet which have the capacity to impact significantly upon research practices within universities. These include changing patterns of collaboration, challenges to accepted notions of intellectual property and copyright, concerns regarding the validation of electronically based information, changes to the time-frames associated with scholarly research, and the respective roles of electronic and hard copy publishing. Each of these areas is of considerable importance in terms of the role of universities in the future.

Special reference is made to a longitudinal study currently being conducted at Australian Catholic University concerning the impact of Internet access on the research practices of academic staff. The study places particular emphasis on identifying and examining possible changes to patterns of collaboration in research and academic writing both within this geographically dispersed, multi-campus institution and with colleagues from elsewhere. Of special interest is the degree to which traditional concepts of 'distance' remain relevant to the activities of the Virtual Campus.
\end{abstract}

\section{Keywords}

Academic writing, Collaboration, Copyright, Communication technology, Research, Virtual campus 


\section{INTRODUCTION}

Recent developments in computing technology, in particular those associated with the Internet, are calling into question a number of the traditions of academic practice. This paper discusses some aspects of the use of the Internet which have the capacity to impact significantly upon research practices within universities. While focussing principally on changing patterns of collaboration, it acknowledges a number of related issues which impinge on the practicalities and consequences of such collaboration within academic contexts, including challenges to accepted notions of intellectual property and copyright, concerns regarding the validation of electronically based information, changes to the time-frames associated with scholarly research, and the respective roles of electronic and hard copy publishing. Each of these areas is significant not simply within the context of particular examples of academic collaboration, but more broadly in terms of the ongoing credibility and authority of universities in relation to the creation, representation and transmission of knowledge, with concomitant implications for the maintenance of the structures which currently support those activities.

In most developed and indeed in many less developed countries across the globe, academics who have access to the technology have embraced with enthusiasm the various facilities and capabilities of the Internet. Email, newsgroups, listservs, MUDs, MOOs, user friendly search engines and the multimedia capacities of the World Wide Web have enticed educators and researchers across most disciplines to engage in a range of new and in many cases significantly different practices. While some of these might be seen as enabling traditional activities to be undertaken with greater ease and efficiency, others actually possess the potential to undermine some of the basic foundations upon which our academic endeavours have traditionally been based. These dimensions are acknowledged already in a range of publications from chapters and articles in volumes such as Changes in Scholarly Communication Patterns (Mulvaney and Steele (eds), 1993), The Cultures of Computing (Star (ed.), 1995) and Work and Technology in Higher Education: the Social Construction of Academic Computing (Shiels (ed.), 1995) through journal articles and conference papers initially emanating from a range of discipline areas, but more recently tending to be concentrated under the conceptual umbrella of the Virtual University or Campus.

\section{THE RESEARCH PROJECT}

In considering these issues, reference is made within this paper to a longitudinal study currently being conducted at Australian Catholic University concerning the impact of Internet access on the research practices of academic staff. The study, currently in its second year, places particular emphasis on identifying and examining possible changes to patterns of collaboration in research and academic writing both within this geographically dispersed, multi-campus institution and with colleagues from other institutions both within Australia and overseas. 
In common with many tertiary institutions, Australian Catholic University is devoting considerable resources to facilitating staff access to the Internet. The benefits are generally presumed to include significantly enhanced opportunities for communication and collaboration with colleagues both within and outside the University in addition to improved access to a range of information sources for research purposes. Communication within the University is of particular interest in that the institution comprises eight campuses, several quite small in size, distributed across three states and one territory of Australia. While the number of staff associated with any one discipline area on a single campus may be extremely small, in some extreme cases consisting of a single individual, these groups are actively encouraged to perceive themselves as colleagues within a single institution, clearly "virtual" in regard to its unity and to a significant extent electronically based, with joint responsibility for the content and in some cases for the teaching of units and courses. Within each of the Faculties of the University, national discipline based networks fulfill many of the functions of traditional academic departments in nurturing and developing intellectual content. Collaboration in research activities is an obvious component of this type of cooperation. The resource implications alone, but also, very importantly, the potential for changes to established practice, suggest the importance of monitoring the extent to which the expectations associated with the implementation of this technology are in fact being met (Dowling, 1996). Of special interest is the degree to which traditional concepts of "distance" can be overcome through a strengthening of electronic infrastructures facilitating virtuality.

The research is being conducted through a series of questionnaires and follow-up interviews. An initial survey directed to all permanent members of the academic staff of the University was used to identify those who had made use of the Internet over the previous 12 months (1995), and who were willing to participate further in the project. Of the 167 responses received by the due date, 103 indicated a willingness to continue their participation. At that stage (mid 1996), 62\% of the 167 respondents were making use of the Internet to communicate with colleagues in relation to academic matters, $42 \%$ specifically in relation to research, while $22 \%$ had used the Internet to write collaboratively with colleagues. When considered in the light of the fact that at that time not all academic staff of the University had computers on their desks, a University based training program in the use of the Internet had been offered only on some campuses, the capacity of the network was inadequate leading to access being somewhat sporadic and there was a dearth of technical support, these figures suggest a considerable willingness to use the technology albeit under less than ideal circumstances.

In relation to the other focus of the project, namely using the Internet to access information, $70 \%$ of the initial respondents had made use of these facilities to look for information related to their teaching, and $68 \%$ for information related specifically to their research activities. While this preliminary questionnaire did not address the issue of overlap between these areas, it is clear that considerable use was being made of the technology for information retrieval for academic purposes. In conversation with a number of respondents it is clear that the fact that these 
percentages were higher than those making use of the Internet for communication and collaboration with colleagues relates to the transparency of the retrieval mechanisms available on the World Wide Web through programs such as Netscape, the University's browser-of-choice.

Of particular influence in developing a rationale and methodology for this project has been a longitudinal investigation undertaken by researchers from the School of Education, Kings College London. This research, funded by the British Library Research and Development Department, has been in progress since 1992. Associated publications to date include Squires (1993), Squires, Barry and Funston (1994), Barry (1995) and Barry and Squires (1995). The Australian Catholic University project is providing a valuable counterpoint to this research, in that the technology which has been provided to staff is significantly more sophisticated and 'user friendly' than that which was initially available to participants in the British study.

\section{COMMUNICATION AND COLLABORATION}

It is undeniable that computer networks have the capacity to encourage and facilitate collaborative research and writing. With the current popular focus on the broader social and economic applications and implications of the Internet, the fact that this was, in fact, one of the primary purposes of its early manifestations, can easily be overlooked. The value of today's enriched communications environment encompassing home pages, email, listservs, discussion groups, MUDs and MOOs, in multiplying the possibilities for communication with colleagues should not be underestimated. However, while applauding the potential for interaction within these environments, it should not be assumed that they are the simple equivalents of more traditional forums including exchanges conducted through the medium of refereed journals, attendance at face to face conferences or other forms of presentation, collaboration within a physically and intellectually delineated context such as a laboratory, communication by telephone or conventional mail or, very importantly, those snatched moments and chance remarks in a corridor or over the coffee dispenser, which may provide a sharply focussed impetus for new research directions or understandings. Each of these situations has its own social complexities which are important in determining outcomes. These are well addressed in relation to electronic environments by writings and research addressing broader aspects of computer mediated interpersonal relationships (Rheingold, 1994; Spender, 1995; Turkle, 1995). Nor should it be assumed that it is necessarily advantageous to replace traditional opportunities for collaboration entirely by the newly available modes of communications. While ongoing developments both in the technology itself and in the use to which it is made by academics render the situation extremely fluid, it might well be that a judicious mix of forms of communication would maximise the benefits to be gained. It has been suggested by several staff interviewed in connection with the Australian Catholic University project that chance encounters of the "coffee dispenser" type 
as distinct from the more purposeful interactions which are initiated on the Internet often have a particularly serendipitous effect on the thinking associated with research activity.

What form does the collaboration of this group of academics take? While the results of the interviews on this subject have not yet been formally analysed, it appears that research related communications cover a range of matters which would be familiar to the traditional academic - namely feedback on ideas for research projects or on written reports and papers, exchanges of information concerning sources of funding and possible outlets for publications, information related to conferences, discussion of the relative merits of other academics as potential supervisors, referees or collaborators, lists of resources pertaining to particular areas of interest and of course the collaborative writing of books, reports, conference papers and the like.

In relation to the search for possible collaborators, it is of interest that several subjects, all younger academics who would not at this stage have had many opportunities for face to face interaction with established researchers in their areas whether in their own country or overseas, alluded to making regular and systematic searches of the home pages of other universities in search of details relating to staff working in the same or allied fields. Having located such people, several of the academics interviewed had gone on to make some form of contact electronically, all of them agreeing that this was less intimidating than the prospect of approaching unknown individuals either in person, on the telephone or ty conventional mail.

A further point of interest which has been noted in interviews with the staff at Australian Catholic University with regard to collaboration is the speed with which the cyberspace within which these interactions take place becomes genuinely divorced from geographical space. While a number of participants in the early interviews expressed special excitement at their new ability to communicate with colleagues overseas, in interviews which occurred only six months later these same academics discussed their interactions, primarily through email, in terms which did not discriminate between the local and the geographically distant. Colleagues at other local campuses, even in offices within the same building, clearly occupied the same notional 'space' as contacts on the other side of the world. This is in keeping with earlier findings (Dowling, 1987) in which the implementation of electronic mail in office buildings of large, dispersed organisations, led to electronic communication taking place indiscriminately between physically distant locations, between adjoining offices, and even between adjoining desks. It is particularly interesting in this context in relation to the conscious goal of the University that it should be both perceived externally and experienced internally as a single, integrated institution. (Other initiatives supporting this aim include extensive use of video conferencing for small group teaching and seminars, and the beginnings of Internet based course offerings between campuses as well as for external students.) 


\section{ACADEMIC WRITING AND THE INTERNET}

Internet access brings with it an enormously enhanced potential for collaborative writing. The ease with which textual material can be exchanged and modified by writers interacting through a range of software types and, if so desired, within a virtually instantaneous timeframe, has for many researchers enabled the realisation of the hitherto somewhat abstract concept of the social construction of knowledge. It also contributes to a possible need to rethink for the future certain issues related to the ownership and distribution of textual material.

Even prior to the widespread availability of networked communication, it could be said that the use of the computer as a writing medium weakened from a number of points of view our usual conceptions concerning the sovereignty of the individual author over text (Dowling, 1995). While many of the psychological and phenomenological subtleties relating to the differences between computer based and other forms of writing are beyond the scope of this paper, there are some obvious considerations which pertain to the electronic composition and dissemination of academic writing.

The very ease with which electronically textual material can be assembled and seamlessly integrated into a single document may raise generalised doubts concerning the source, the originality and the uniqueness of a particular piece of work or of its component parts. The possibilities for such involuntary 'collaboration' are exacerbated by the extent to which current developments in communications technology, particularly those related to the Internet, facilitate the uncontrolled dissemination of material previously subject to processes of distribution which enabled the source to be acknowledged and validated. For the academic community, dependent as it has traditionally been on the notion of the authoritative text for which an identifiable individual author or authors may be held responsible, such developments raise a number some dilemmas. A recurrent theme in discussion of this issue with participants in the Australian Catholic University study has been the need to balance the advantages of electronic dissemination of research related discussion and findings with the increased risks that such readily accessible material may be taken and re-used without due acknowledgment. Many academics, including this author, can already provide instances of such practice. While plagiarism is not, of course, a new problem, the ready availability of large amounts of material in "useable" form greatly facilitates its occurrence. In addition, the sheer volume of information available on the Internet decreases the likelihood of detection in that few academics can now claim intimate knowledge of all, or even most of the texts available in their field. Indeed in both the King's College (Barry and Squires, 1995) and the Australian Catholic University studies, researchers have expressed an ongoing need for training in techniques of information retrieval in order to cope with the ever increasing amount of material available electronically. 


\section{ACADEMIC PUBLISHING AND THE LEGITIMACY OF ACADEMIC KNOWLEDGE}

Within academic contexts, the contrast between the potential for rapid electronic dissemination and the painstaking but often frustratingly slow process of publishing in refereed academic journals has led in many disciplines to a mixture of publishing media becoming legitimised by default, for different purposes. Where speed of dissemination of research findings is seen as important, particularly in the more "practically" oriented disciplines such as many of the sciences, it is now widely accepted that the "real" exchanges of ideas and results takes place through electronic media, with publication in traditional paper based journals increasingly becoming a retrospective event, having more to do with the formal recognition of achievements and credentials associated with funding and promotion processes than with the actual process of the creation and dissemination of knowledge. It is interesting to note in this regard the recent burgeoning of electronic 'journals' subject to refereeing processes which compare favourably with those associated with traditional paper based journals endorsed by the academic hierarchies. In some instances the hard copy and electronic versions of the same publication complement one another while fulfilling different needs.

Traditional understandings of the legitimacy of academic knowledge depend heavily on processes of validation through which sources of information may be established and their 'legitimacy' assessed. In the realms of tradit:onal publishing, methods of ascertaining and evaluating the source of textual material included the reputation of the publishing body, particularly in regard to the processes by which a manuscript was accepted for publication, a clear understanding of the extent to which the purported author was in fact responsible for both the content and the presentation of the text and so on. In the case of electronic publishing, the 'provenance' of information is less apparent, putting far more of the onus of evaluation on the individual reader. While this is very much more in keeping with the processes which apply outside academia, it lies at a considerable remove from the well established scaffolding of citation and counter citation which has traditionally supported the structures of 'legitimate' academic knowledge. The extent to which this is perceived as a problem is in part a function of our philosophical beliefs concerning the nature of 'knowledge', in particular the extent to which we are comfortable with the notion of a degree of 'social' construction which includes participants who may be situated well outside the boundaries delineating those traditionally accepted as 'knowledgeable'.

Understandably much of the academic hierarchy, including funding bodies, approaches the issue of non-traditional forms of publication with considerable caution. There is certainly wide variation in the processes which govern electronic publication, ranging from the totally unregulated output of an individual to stringent systems of quality control which equate quite reasonably with traditional practice. With time, the differences between these should become more widely understood. However while in some fields there is an obvious need for recognised means of attesting to the accuracy of published material, in other areas it might be 
suspected that the anxieties have more to do with such issues as the establishment of status both within and between disciplines, or the maintenance of existing systems of promotion, than with concerns about knowledge per se.

This is an area which currently generates heated debate among the Australian Catholic University staff interviewed, both in relation to their own research and to the standards which should govern the use and citation of electronically based sources by students. There is clear variation between disciplines and between individuals within disciplines. Within this University there is pressure from individual staff in a range of areas, including both the sciences and the humanities, for a cautious acceptance of the new publishing media - including the Internet and CD ROM or disk based publications. Considerable resentment is expressed at the perceived intransigence of some elements in the academic hierarchy which do not as yet afford great value to such publications.

Of course the sheer availability of academic resources via the Internet creates other consequences in relation to knowledge creation, including concerns as to whether the timeframes traditionally associated with research degrees should be shortened in acknowledgment of the benefits both of more efficient information retrieval and of the efficacious effects of word processing on academic writing. While acceleration of the research process might be perceived as having benefits in terms of efficiency and economic considerations, some of the Australian Catholic University academics have expressed concern that this could result in insufficient "thinking" time being available to researchers, with deleterious consequences for research outcomes. Similar concerns were voiced by researchers interviewed during the Kings College study (Barry and Squires, 1995).

\section{COPYRIGHT}

Matters related to the definition and regulation of intellectual property, including basic copyright law, are obviously of special interest to academics. Within the context of Internet based research and publication, questions relating to the ownership of electronically mediated textual material are particularly important.

In addressing these issues we tend to assume that, for the majority of people who may be involved, traditional understandings of individual ownership and of property rights related to the control of intellectual assets remain basically unchanged. While the widely touted concept of the computer based information society has undoubtedly strengthened public acceptance of the notion of intangible products such as "information" as tradable commodities, it is possible that the extraordinary burgeoning of a proliferation of alternative network 'cultures' might stimulate broader adherence to a number of contrary points of view upon which ethical or moral decision making could conceivably be based in the future, including the idea that information should be freely accessible to all. Such beliefs are clearly at odds both with traditional understandings and with the growing emphasis on the commodification of abstract information. 
Both individual academics and their institutions require clarity in relation to the ownership of the products of research activity. While patent law is obviously of great importance in many fields, the potential for change wrought by computing technology pertains most strongly to questions of copyright. Much of the discussion concerning the legal aspects of ownership of computer based material centers around the abstract or ephemeral form of the electronically stored product as compared with the fixed and tangible nature of a paper based document. As a consequence of such qualities, identification of 'original' text or of subsequent alterations may not be possible. Further, the new ease of distribution through computer networks, allied with ideological positions supporting so-called 'free' access to computer based information, introduces complexities which may not be adequately addressed through piecemeal modifications to existing law. It is undeniable that the supreme ease with which electronically based material may be copied, modified and disseminated without the consent of the original author creates significant challenges to existing copyright regulations, a number of which are currently under debate around the world.

This uncertainty is an obvious factor in the hesitation felt at both an individual and a systemic level regarding the status of electronic publications.

\section{CONCLUSION}

The embracing of the Internet and associated technologies by academics is inevitable and clearly has many benefits to offer, particularly in relation to the ability to access information and to communicate with colleagues. Nevertheless, as argued in this paper, there are a number of issues associated with the use of this technology of which researchers themselves and those responsible for the encouragement and management of research should be aware. There is currently a great deal of interest, at both a theoretical and a practical level, in the use of the Internet for teaching and learning. However there is also a strong sense in which the research enterprise underpins the whole notion of a university, whether virtual or otherwise. This aspect should not be neglected in our consideration of the impact of computing and communications technology on tertiary institutions.

\section{REFERENCES}

Barry, C. (1995) Critical issues in evaluating the impact of IT on information activity in academic research: developing a qualitative research solution, in Library and Information Science Research, 17 (2), pp. 107 - 134.

Barry, C. and Squires, D. (1995) Why the move from traditional informationseeking to the electronic library is not straightforward for academic users: some surprising findings, in Online Information 95, Meckler Press, London, pp. $177-187$. 
Dowling, C. (1987) Mind Your Language: Computers and Communication, in Proceedings of the Australian Computers in Education Conference, Hancock, J. (ed.), Adelaide 1987, pp. 39 - 43.

Dowling, C. (1995) Quills to keyboards: the impact of computing technology on academic writing, in Proceedings of the 1995 Annual Conference of the Higher Education and Research Development Society of Australasia (HERDSA), Rockhampton, July 1995, pp. 270 - 276.

Dowling, C. (1996) Whose words are they? Some aspects of the ownership of textual material in electronic environments, in Proceedings of the Australian Computers in Education Conference, (disk format) Canberra, April 1996.

Dowling, C. (1996) Academia Online: the impact of the Internet on academic research practices, in Proceedings of the 1996 Annual Conference of the Higher Education and Research Development Society of Australasia (HERDSA), Perth, July 1996.

Fraser, M. (1993) Intellectual Property in Australia: Libraries and Copyright, in Mulvaney, J. and Steele, C (eds) (1993), Changes in Scholarly Communication Patterns, Canberra: Australian Academy of the Humanities, Occasional Paper No. 15.

Lyman, P. (1993) Libraries, Publishing and Higher Education, in Mulvaney, J. and Steele, C (eds) (1993), Changes in Scholarly Communication Patterns, Australian Academy of the Humanities, Canberra, Occasional Paper No. 15.

Mulvaney, J. and Steele, C (eds) (1993) Changes in Scholarly Communication Patterns, Australian Academy of the Humanities, Canberra, Occasional Paper No. 15.

Rheingold, H. (1994) The Virtual Community. Secker and Warburg, London.

Shiels, M. (ed.) (1995), Work and Technology in Higher Education: the Social Construction of Academic Computing. Lawrence Erlbaum, New Jersey.

Spender, D. (1995) Nattering on the Net: Women, Power and Cyberspace. Spinifex Press, North Melbourne.

Squires, D. (1993) The Use of Information Technology to Support Information Access in Research, in D. Johnson and B. Samways (eds), Informatics and Changes in Learning, Elsevier Science Publishers B.V., Amsterdam, pp. 183 185.

Squires, D., Barry, C. and Funston, T. (1994) The Use of IT-Assisted Information Systems in Academic Research. British Library Research and Development Department, London, Report No. 6215.

Star, S.L. (ed.) (1995) The Cultures of Computing. Sociological Review Monograph, Blackwell, Oxford.

Turkle, S. (1995) Life on the Screen: Identity in the Age of the Internet. Simon and Schuster, New York. 


\section{BIOGRAPHY}

Carolyn Dowling received a $\mathrm{PhD}$ from Monash University, Melbourne, Australia in 1994. A lecturer in computing since 1984, she is currently an Associate Professor at the Australian Catholic University. She is Head of the School of Arts and Sciences, which encompasses the Department of Mathematics, Science and Information Technology, the Department of Humanities and the Department of Visual and Performing Arts. Her research interests include a range of $\mathrm{HCl}$ issues, the impact of the Internet on academic research practices, 'virtual' environments for learning, and computer based writing. 